\title{
Central Nervous System Involvement is Associated with Ibrutinib-Induced Severe Hyperleukocytosis and Controlled by the Combination of Intrathecal Chemotherapy in Chronic Lymphocytic Leukemia: A Case Report
}

\author{
Ke Wang, Zheng Yan, Junfeng Chu, Shuang Zhao, Haiying Wang, Yanyan Liu* \\ Department of Internal Medicine, Affiliated Cancer Hospital of \\ Zhengzhou University \& Henan Cancer Hospital, Zhengzhou, \\ ${ }^{*}$ Corresponding authors \\ Henan, China \\ Yanyan Liu, Department of Internal Medicine, Affiliated Cancer Hospital \\ of Zhengzhou University \& Henan Cancer Hospital, 127 Dong Ming Road, \\ Zhengzhou, Henan 450008, China, email: yyliu@zzu.edu.cn
}

Submitted: 29 March2021; Accepted: 07 Apr 2021;Published: 14 Apr 2021

Citation: Ke Wang, Zheng Yan, Junfeng Chu, Shuang Zhao, Haiying Wang, et al. (2021) Central Nervous System Involvement is Associated with Ibrutinib-Induced Severe Hyperleukocytosis and Controlled by the Combination of Intrathecal Chemotherapy in Chronic Lymphocytic Leukemia: A Case Report. Medical \& Clinical Research 6(4): 506-509.

\begin{abstract}
Central nervous system involvement (CNSi) is a rare complication of chronic lymphocytic leukemia (CLL) with a very poor prognosis. Its diagnosis, predictive factors, and standard treatment remain elusive. Ibrutinib, an oral inhibitor of Bruton's tyrosine kinase, has been approved to treat CLL, yet little is known of its effect on the occurrence and management of CNSi. Here, we report, for the first time, an elderly CLL patient with secondary CNSi following ibrutinib-induced severe hyperleukocytosis, characterized by subcutaneous lesions and unmutated IgHV gene. Ibrutinib combined with intrathecal chemotherapy induced a prompt and durable remission of CNSi. This case indicates that severe hyperleukocytosis caused by ibrutinib is associated with the emergence of CNSi, and a combined treatment with intrathecal chemotherapy is effective and safe in the treatment of CLL patient with CNSi.
\end{abstract}

Keywords: Chronic Lymphocytic Leukemia, Central Nervous System Involvement, Ibrutinib, Intrathecal Chemotherapy

\section{Abbreviations:}

CNSI: Central Nervous System Involvement;

CLL: Chronic Lymphocytic Leukemia;

WBC: White Blood Cell;

CSF: Cerebrospinal Fluid;

CR: Complete Remission;

AHF: Acute Heart Failure

\section{Introduction}

Chronic lymphocytic leukemia (CLL) is a malignant clonal disorder of B lymphocytes, characterized by the proliferation and accumulation of mature small lymphocytes in the blood, bone marrow, and lymphoid tissues [1]. Even though CLL is the most common type of leukemia in adults, the prevalence of central nervous system involvement (CNSi) in CLL is very low $(\sim 0.8 \%)$ [2]. However, the clinical manifestations of CNSi in CLL are heterogeneous and do not always appear. Therefore, immunophenotyping of cerebrospinal fluid (CSF) is more important for the diagnosis of CNSi in CLL than neuroimaging. Moreover, there are no clear treatment guidelines for CLL with CNSi. Ibrutinib is an oral inhibitor of Bruton's tyrosine kinase that has been approved as first-line treatment for CLL. Herein, we reported an elderly CLL patient with secondary CNSi for
54 months, presenting with peculiar subcutaneous lesions, unfavorable unmutated IgHV, and severe hyperleukocytosis after initiation of ibrutinib treatment, but the patient achieved a prompt durable remission of $\mathrm{CNSi}$ with the combination of intrathecal chemotherapy and ibrutinib.

\section{Case Presentation}

A 70-year-old female was noticed to have a high white blood cell (WBC) count of $28 \times 109 / \mathrm{L}$ during a routine physical examination in January 2015. Subsequent bone marrow biopsy and flow cytometry assay detected abnormal B-cell clones. Bone marrow aspiration showed $61.5 \%$ mature lymphocytes, and flow cytometric analysis revealed $51.5 \%$ of monoclonal B cells expressing CD5, CD19, HLA-DR, CD20, and restricted kappa light chain. Multiple swollen lymph nodes with a diameter of $<2 \mathrm{~cm}$ were observed. Based on these clinical examinations, the patient was diagnosed with CLL. She had no symptoms at the time of diagnosis. The patient's medical history included coronary heart disease, hypertension, and chronic obstructive pulmonary disease. Thus, a watch and wait approach was selected for her.

She was admitted to our hospital again due to grown subcutaneous masses in her head and neck in December 2017. Laboratory tests 
showed elevated blood cell counts and biochemical parameters, including WBC $106.42 \times 109 / \mathrm{L}$, lymphocytes $93.07 \times 109 / \mathrm{L}$, platelets $143 \times 109 / \mathrm{L}$, hemoglobin $114 \mathrm{~g} / \mathrm{L}$, lactate dehydrogenase $294 \mathrm{U} / \mathrm{L}$ (normal 120-249 U/L), and $\beta 2$-microglobulin 3.4 $\mathrm{mg} / \mathrm{L}$ (normal 1.0-2.3 $\mathrm{mg} / \mathrm{L}$ ). Positron emission tomography and computed tomography (PET/CT) scan revealed small subcutaneous masses in the head and neck, bilateral enlargement in the cervical, supraclavicular, and axillary lymph nodes, bilateral lymphadenopathy in the groin, mediastinum, abdominal cavity, and retroperitoneum with the longest diameter of $32 \mathrm{~mm}$ and SUV max 6.6, and splenomegaly with the SUV max 3.7 (Figure 1A and 1B). An enlarged lymph node was completely resected for pathological evaluation, which indicated small lymphocytic lymphoma.

Wang et al. Figure 1
(A)

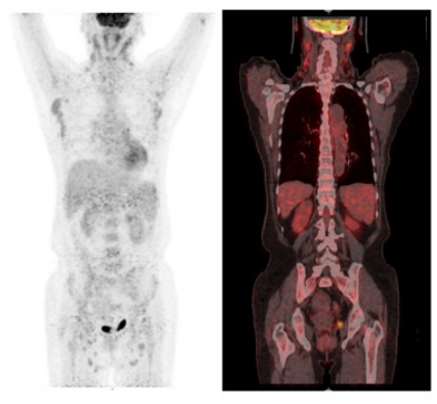

(B)

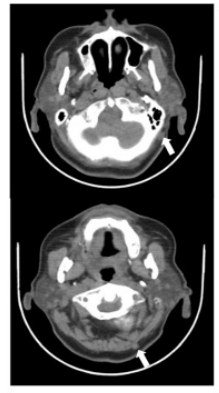

Peripheral blood and bone marrow cytology showed an increase in mature small lymphocytes (Figure 2A). Flow cytometry analysis of bone marrow cells showed that $70 \%$ of mature small lymphocytes were abnormal, with the expression of CD5, CD19, CD43, CD200, CD23, and Kappa and partial expression of CD20 and CD22 (dim), but no expression of CD10, CD79b, FMC-7, Lambda, CD38, CD103, CD3, CD4, CD8, and CD56 (Figure 2B). Fluorescence in situ hybridization (FISH) assay indicated that CCND1, P53, RB-1, ATM, CSP12, and D13S25 were all negative. This case met the iwCLL diagnosis criteria for CLL with Rai stage II and Binet B. Then, she received six cycles of rituximab plus chlorambucil from December 2017 to May 2018, and achieved a complete remission (CR) according to the iwCLL guidelines for the response assessment of CLL [3]. Flow cytometry analysis showed negative for abnormal bone marrow and peripheral blood lymphocytes. Whole-body CT scan demonstrated normal lymph nodes and spleen in size and the disappearance of subcutaneous masses. Moreover, her complete blood count fell into the normal range. Thereafter, oral chlorambucil was given as maintenance treatment at the same dose.
Wang et al. Figure 2

(A)

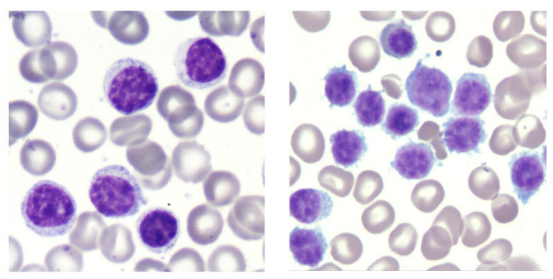

(B)
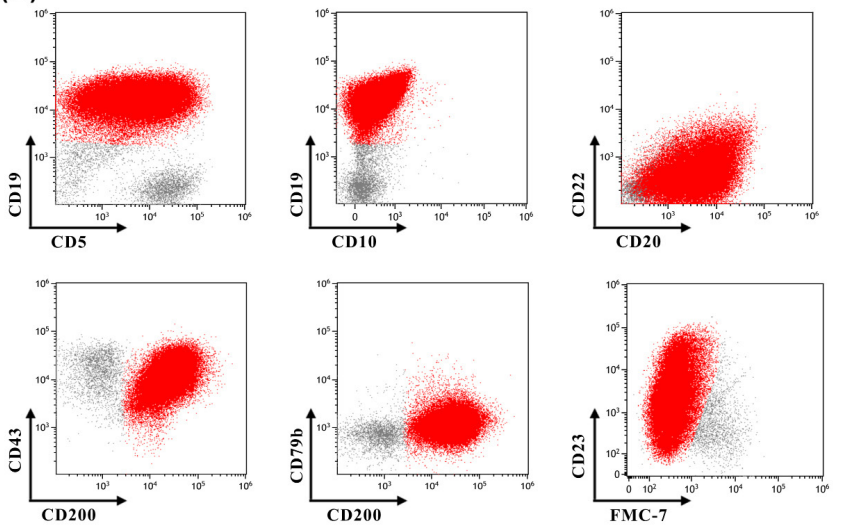

In July 2019, the patient began to suffer from eye swelling, abdominal distension, and weakness. WBC count was $51.72 \times$ 109/L and platelet count $58 \times 109 / \mathrm{L}$. CT scan showed progression of disease with multiple enlarged lymph nodes. Bone marrow flow cytometry analysis confirmed the relapse of CLL. Gene mutation assay indicated that the IGHV gene was unmutated and there were no other genetic aberrations. She started oral ibrutinib with a daily dose of $420 \mathrm{mg}$. After ibrutinib treatment for 20 days and shortterm improvement of symptoms, the patient was hospitalized due to severe fatigue, slurred speech, and decreased consciousness. She was afebrile, and the results of neurological examination showed no abnormalities. CT scan revealed a partial remission of enlarged lymph nodes. The total WBC count increased to $193.7 \times$ $109 / \mathrm{L}$, mainly lymphocytes, and platelet count rose to $64 \times 109 / \mathrm{L}$. A brain MRI scan was performed to rule out cerebral hemorrhage and infarction. After excluding infectious diseases, meningeal metastasis was confirmed by CSF examination. The CSF was clear and its biochemical analysis results revealed normal glucose, elevated WBC count $(0.3 \times 109 / \mathrm{L})$, elevated protein levels $(0.811$ 
$\mathrm{g} / \mathrm{L})$, and slightly low chlorine (118.5 $\mathrm{mmol} / \mathrm{L})$. CSF cytology showed that a proportion of lymphocytes were abnormal, but blood contamination was ruled out (Figure 3A). The CSF flow cytometric analysis indicated that abnormal monoclonal B lymphocytes accounted for $67.52 \%$ of all nucleated cells with positive CD5, CD19, CD20, and Kappa and negative CD10 and Lambda (Figure 3B).

(A)
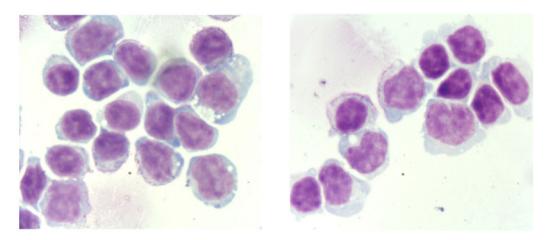

(B)
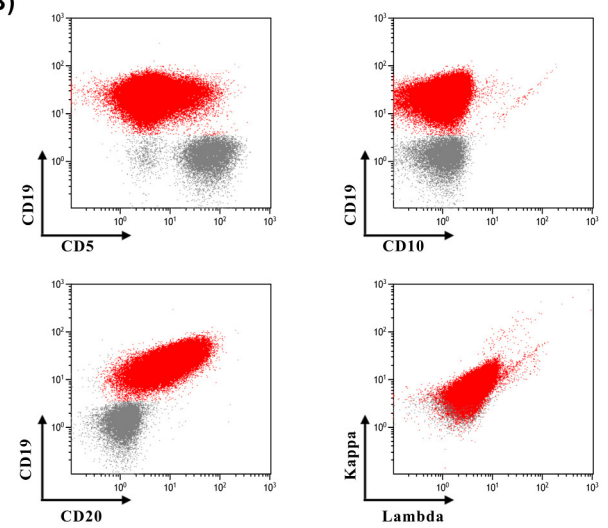

Since ibrutinib induced partial remission of lymph node enlargement, it was continued as first-line treatment. However, intrathecal chemotherapy (methotrexate $15 \mathrm{mg}$, cytarabine 50 $\mathrm{mg}$, and dexamethasone $5 \mathrm{mg}$, twice a week) was added to the targeted therapy. After the completion of four administrations of intrathecal chemotherapy, abnormal B lymphocytes in CSF were completely eliminated and CNSi symptoms disappeared. Thus, the patient stopped intrathecal chemotherapy but continued to receive ibrutinib monotherapy. Nevertheless, ibrutinib was discontinued two weeks later because the patient had grade IV acute heart failure (AHF). ST-segment changes were found in the electrocardiograph, and blood gas analysis suggested a decrease in the partial pressure of oxygen. It was observed that plasma troponin I levels increased to $0.3 \mu \mathrm{g} / \mathrm{L}$, and $\mathrm{N}$-terminal pro-brain natriuretic peptide concentrations were as high as $14,768 \mathrm{pg} / \mathrm{L}$. After cardiac function returned to grade I, Ibrutinib was restarted daily at a reduced dose of $140 \mathrm{mg}$. During her follow-up visit in February 2020, the patient did not experience any cardiac discomfort with this reduced dose of ibrutinib and CLL was well managed. In addition, there was no generalized lymphadenopathy again, WBC count was approximately $19 \times 109 / \mathrm{L}$ and platelet count was about $70 \times 109 / \mathrm{L}$. Unfortunately, the patient was admitted to the hospital due to lung infection and eventually died of multiple organ failure on April 3, 2020.

\section{Discussion}

CNSi of CLL usually presents with non-specific symptoms, such as headaches, changes in mental status, cranial nerve abnormalities, optic neuropathy, lower limb weakness, and cerebellar signs. Thus, a differential diagnosis is needed to help diagnose CNSi of CLL. In this case study, brain MRI scan failed to find abnormalities and rule out cerebral hemorrhage and infarction. In contrast, CSF examination had a key role in the diagnosis, especially by flow cytometric analysis. In CSF, no red blood cells were observed, indicative of no blood contamination. Also, the results of CSF biochemical analysis ruled out the possibility of infectious disease. When the combination of intrathecal chemotherapy and ibrutinib achieved a rapid disappearance of neurological symptoms and clearance of CLL cells in CSF, lymphocytosis was persistent during the CNSi treatment. All of these findings ruled out the possibility of blood contamination in CSF.

So far, there are no high-risk factors that can be used to predict CNSi in CLL patients, including the Rai stage, disease progression and duration, prior CLL therapy, IGHV mutational status, ZAP-70, FISH, and CD49d $[2,4]$. When it comes to this CLL patient with secondary CNSi, she presented with peculiar subcutaneous masses, unfavorable unmutated IGHV, and severe hyperleukocytosis. In recent years, CLL treatment has been revolutionized with the advent of the inhibitors of B-cell receptor signal transduction, such as ibrutinib. It has been reported that ibrutinib may overcome the poor prognosis of CLL patients with unmutated IGHV genes. Indeed, in this case ibrutinib induced obvious shrinkage of enlarged lymph nodes. Disappointedly, CNSi appeared within 20 days after of initiation of ibrutinib treatment, accompanied by severe hyperleukocytosis. As the most common adverse event in the early phase of treatment, the association of ibrutinib-induced severe hyperleukocytosis with CNSi of CLL is not clear. Previous studies have suggested that a large number of leukemia cells in the peripheral blood can increase the risk for CNSi through simple perfusion and extravasation [5]. Hyperleukocytosis has been recognized as a risk factor for CNSi in the development of acute myelogenous leukemia and acute lymphocytic leukemia [6]. Although the mechanisms by which leukemia cells infiltrate the brain remains unclear, leukemia cells can gain access to this compartment in different ways: transmigrating into the subarachnoid space via the perforated cerebral vessels; entering the CSF from the seeded meninges, especially via the ependymal lining and choroid plexus; and invading the central nervous system via perineural sheaths on cranial and spinal nerve roots [7]. Therefore, it is necessary to closely monitor the emergence of CNSi in CLL patients when ibrutinib-induced severe hyperleukocytosis occurs.

Due to relatively few cases of CLL patients with CNSi reported so far, no proven treatment guidelines for these patients are available. Traditionally, limited treatment options include radiotherapy and intrathecal chemotherapy with or without high-dose intravenous methotrexate. Recently, ibrutinib has been shown to be capable of crossing the blood-brain barrier and achieve clinically significant concentration in the CSF [8]. Tam presented a case of CLL with CNSi that responded promptly to ibrutinib monotherapy and no recurrence after eighteen months [9]. In a small retrospective study that documented four CLL patients with CNSi undergoing monotherapy with ibrutinib, all of them achieved CSF clearance within three months, three of them achieved CR, and no relapse was found after a median follow-up of nine months [10]. In 
another retrospective cohort of CLL patients with CNSi, all six patients receiving ibrutinib achieved response with $3 \mathrm{CR}$ and 3 $\mathrm{PR}$, including patients with $17 \mathrm{p}$ deletion and refractory/relapsed disease [11]. Here, we report for the first time a definitive CNSi of CLL secondary to ibrutinib-induced severe hyperleukocytosis. For this case, after diagnosis of leptomeningeal disease, intrathecal chemotherapy was added to the treatment regimen. Actually, this combination treatment regimen not only quickly eliminated meningeal metastasis, but also achieved durable remission for nearly nine months. The results indicate that ibrutinib combined with intrathecal chemotherapy is effective and safe for the CLL patients with CNSi. Unfortunately, this patient experienced a dose reduction due to AHF and died of infection-induced multiple organ failure eventually. Thus, the toxicity of ibrutinib is still worthy of attention. According to reports, common adverse events of ibrutinib include atrial arrhythmias, infection, bleeding, skin rash, joint pain, and diarrhea [12]. Interestingly, a research team has used the VigiBase to identify ibrutinib-associated mortality, including supraventricular arrhythmias, central nervous system hemorrhagic events, heart failure, ventricular arrhythmias, conduction disorders, ischemic stroke, and hypertension-related organ damage, which usually appeared earlier [13, 14]. The mechanism behind the adverse effects of ibrutinib is mediated by off-target inhibition, such as the inhibition of phosphoinositide 3-kinase [15]. Studies have shown that pre-existing diabetes, hypertension, and prior history of heart disease are important risk factors for therapy-related mortality [16]. It is recommended to conduct a comprehensive assessment of the clinical benefits and risks before using ibrutinib.

In summary, this case shows that severe hyperleukocytosis caused by ibrutinib may accelerate the occurrence of CNSi in CLL patients, and the addition of intrathecal chemotherapy to the targeted therapy is effective and safe in the rapid remission of the complication of CLL. In addition, the importance of early use and toxicity management of ibrutinib is emphasized.

\section{Ethics approval}

The approval has been obtained to publish the case details from the Institutional Review Board of the Affiliated Cancer Hospital of Zhengzhou University, and written informed consent has been provided by the patient's next of kin to have the case details and any accompanying images published.

\section{Acknowledgments}

The authors thank Xiaojiao Wang of Central Laboratory for her support of bone marrow and CSF extraction. We thank the patient's family members for their consent to publish the case details.

\section{ORCID}

Ke Wang ID https://orcid.org/0000-0001-9585-7944

Zheng Yan ID https://orcid.org/0000-0001-9324-2161

Yanyan Liu ID https://orcid.org/0000-0002-3887-0840

\section{References}

1. Chiorazzi N, Rai K, Ferrarini M (2005) Chronic lymphocytic leukemia. The New England journal of medicine 352: 804815.

2. Strati P, Uhm JH, Kaufmann TJ (2016) Prevalence and characteristics of central nervous system involvement by chronic lymphocytic leukemia. Haematologica 101: 458-465.

3. Hallek M, Cheson B, Catovsky D (2018) iwCLL guidelines for diagnosis, indications for treatment, response assessment, and supportive management of CLL. Blood 131: 2745-2760.

4. Wang M, Shih L, Dunn P, Kuo M (2000) Meningeal involvement in B-cell chronic lymphocytic leukemia: report of two cases. Journal of the Formosan Medical Association = Taiwan yi zhi 99: 775-778.

5. Hoffman M, Valderrama E, Fuchs A, Friedman M, Rai K (1995) Leukemic meningitis in B-cell prolymphocytic leukemia. A clinical, pathologic, and ultrastructural case study and a review of the literature. Cancer 75: 1100-1103.

6. Lazarus HM, Richards SM, Chopra R (2006) Central nervous system involvement in adult acute lymphoblastic leukemia at diagnosis: results from the international ALL trial MRC UKALL XII/ECOG E2993. Blood 108: 465-472.

7. Cramer S, Glaspy J, Efird J, Louis D (1996) Chronic lymphocytic leukemia and the central nervous system: a clinical and pathological study. Neurology 46: 19-25.

8. Lionakis MS, Dunleavy K, Roschewski M (2017) Inhibition of B Cell Receptor Signaling by Ibrutinib in Primary CNS Lymphoma. Cancer Cell 31: 833-843.

9. Tam CS, Kimber T, Seymour JF (2017) Ibrutinib monotherapy as effective treatment of central nervous system involvement by chronic lymphocytic leukaemia. Br J Haematol 176: 829831.

10. Wanquet A, Birsen R, Lemal R (2016) Ibrutinib responsive central nervous system involvement in chronic lymphocytic leukemia. Blood 127: 2356-2358.

11. Wanquet A, Birsen R, Bonnet C (2017) Management of central nervous system involvement in chronic lymphocytic leukaemia: a retrospective cohort of 30 patients. Br J Haematol 176: 37-49.

12. Mato AR, Nabhan C, Thompson MC (2018) Toxicities and outcomes of 616 ibrutinib-treated patients in the United States: a real-world analysis. Haematologica 103: 874-879.

13. Salem JE, Manouchehri A, Bretagne M (2019) Cardiovascular Toxicities Associated with Ibrutinib. J Am Coll Cardiol 74: 1667-1678.

14. Woyach JA, Ruppert AS, Heerema NA (2018) Ibrutinib Regimens versus Chemoimmunotherapy in Older Patients with Untreated CLL. N Engl J Med 379: 2517-2528.

15. Stephens DM, Byrd JC (2019) How I manage ibrutinib intolerance and complications in patients with chronic lymphocytic leukemia. Blood 133: 1298-1307.

16. Wiczer TE, Levine LB, Brumbaugh J (2017) Cumulative incidence, risk factors, and management of atrial fibrillation in patients receiving ibrutinib. Blood Adv 1: 1739-1748.

Copyright: (02021 Yanyan Liu, et al. This is an open-access article distributed under the terms of the Creative Commons Attribution License, which permits unrestricted use, distribution, and reproduction in any medium, provided the original author and source are credited. 